\title{
CARACTERIZACIÓN DE PRODUCCIÓN DE ETANOL A PARTIR DE ZACATE BUFFEL Y SUS IMPLICACIONES AMBIENTALES
}

\author{
Characterization of ethanol production from buffelgrass \\ and their environmental implications
}

EPISTEMUS

ISSN: 2007-8196 (electrónico)

ISSN: 2007-4530 (impresa)

Kareen Krizzan Encinas Soto ${ }^{1}$

Abraham Rogelio Martin García ${ }^{2}$

Manuel Pérez Tello ${ }^{3}$

Recibido: 12 de septiembre de 2016,

Aceptado: 30 de noviembre del 2016

Autor de Correspondencia:

Dra. Kareen Krizzan Encinas Soto

Correo: kareen.encinas@iq.uson.mx

\section{Resumen}

En esta investigación se caracterizó el potencial para la obtención de etanol a partir de la planta silvestre Cenchrus ciliaris, también conocida como zacate buffel. A partir de estudios experimentales a nivel laboratorio, se determinaron los rendimientos técnicos y requerimientos energéticos de las etapas de pretratamiento ácido, hidrólisis enzimática y fermentación. Los valores obtenidos fueron comparados con los reportados en la literatura para otros procesos de producción de combustibles. Los resultados del presente trabajo indican que el procesamiento de Cenchrus ciliaris consume la tercera parte de la energía requerida para procesar gasolina por $1 \mathrm{MJ}$ de etanol producido. Asimismo, mediante este proceso es factible la reducción de las emisiones de gases de efecto invernadero por $1 \mathrm{MJ}$ de energía de etanol producido en aproximadamente $40 \%$ su valor actual.

Palabras clave: Hemicelulosa, celulosa, hidrólisis ácida.

\begin{abstract}
In this research, the ethanol production from the wild plant Cenchrus ciliaris, also known as buffelgrass, was characterized. The technical performance and energy requirements for the steps of acid pretreatment, enzymatic hydrolysis and fermentation were determined from experimental studies under laboratory conditions. The values obtained were compared with those reported in the literature for other fuel production processes. The results of this work show that the production of $1 \mathrm{MJ}$ of ethanol from Cenchrus ciliaris requires about one third of the energy necessary to produce $1 \mathrm{MJ}$ of gasoline. Also, the process is capable of reducing by $40 \%$ the emissions of greenhouse gases regarding the production of $1 \mathrm{MJ}$ of ethanol.
\end{abstract}

Key words: Hemicellulose, cellulose, acid hydrolysis.

1 Departamento de Ingeniería Química y Metalurgia, Universidad de Sonora/Correo: kareen.encinas@iq.uson.mx

2 Departamento de Ingeniería Química y Metalurgia, Universidad de Sonora/Correo:armartin@iq.uson.mx

3 Departamento de Ingeniería Química y Metalurgia, Universidad de Sonora/Correo: mperezt@iq.uson.mx 


\section{INTRODUCCIÓN}

El etanol es el biocombustible más utilizado para el transporte en todo el mundo. La biomasa lignocelulósica puede ser una alternativa para producir etanol. La importancia del bioetanol ha ido en aumento principalmente por razones como el calentamiento global y el cambio climático.

La producción de etanol del mundo fue de $31 \mathrm{mil}$ millones de litros en el 2001, la cual incrementó a 39 mil millones de litros en el 2006 [1], y se espera llegar a los 140 billones de litros en 2017. La producción de etanol a partir de materiales lignocelulósicos ha incrementado los últimos años. Lo anterior, es debido a que la mayoría de los materiales lignocelulósicos son renovables, de bajo costo y se encuentran disponibles en abundancia. Algunos de estos materiales incluyen de residuos de cultivos, pastos, aserrín, madera, entre otros [2]. Se ha estimado que 442 mil millones de litros de etanol pueden ser producidos a partir de biomasa lignocelulósica, y que los cultivos totales y residuos de los cultivos pueden producir 491 mil millones de litros de etanol por año, alrededor de 16 veces más que la producción actual de etanol en el mundo [2].

La producción de etanol a partir de biomasa lignocelulósica comprende las siguientes etapas principales: pretratamiento, hidrólisis enzimática y fermentación/destilación/separación. El pretratamiento con ácido diluido es el proceso más eficaz para materiales lignocelulósicos, porque genera cantidades más bajas de productos de degradación que los pretratamientos con ácido concentrado [4,5], lo que es favorable para el proceso de fermentación. La hidrólisis enzimática puede realizarse a los tejidos pretratados de la etapa anterior, las cuales son susceptibles a ser degradados por enzimas hidrolíticas. La aplicación de enzimas hidrolíticas en la producción de etanol consiste en el uso de hemicelulasas y celulasas. Las hemicelulasas pueden ser una alternativa para el pretratamiento por hidrólisis ácida, las celulasas son utilizadas para degradar la celulosa cuando la hemicelulosa es hidrolizada. El etanol puede obtenerse a partir de la xilosa obtenida por hidrólisis de hemicelulosa y de la glucosa obtenida por hidrólisis de celulosa y hemicelulosa. La fermentación de glucosa a etanol es realizada por Saccharomyces cerevisiae, mientras que la fermentación de xilosa es realizada por otros organismos diferentes como son Candida shehatae y Pichia stiptis. La fermentación alcohólica puede llevarse a cabo por lotes o en forma continua. El producto de la fermentación, se somete a un proceso de purificación. Para poder utilizar el etanol como combustible mezclándolo con gasolina, es necesario eliminar el agua hasta alcanzar una pureza del 99.5 al $99.9 \%$ vol.

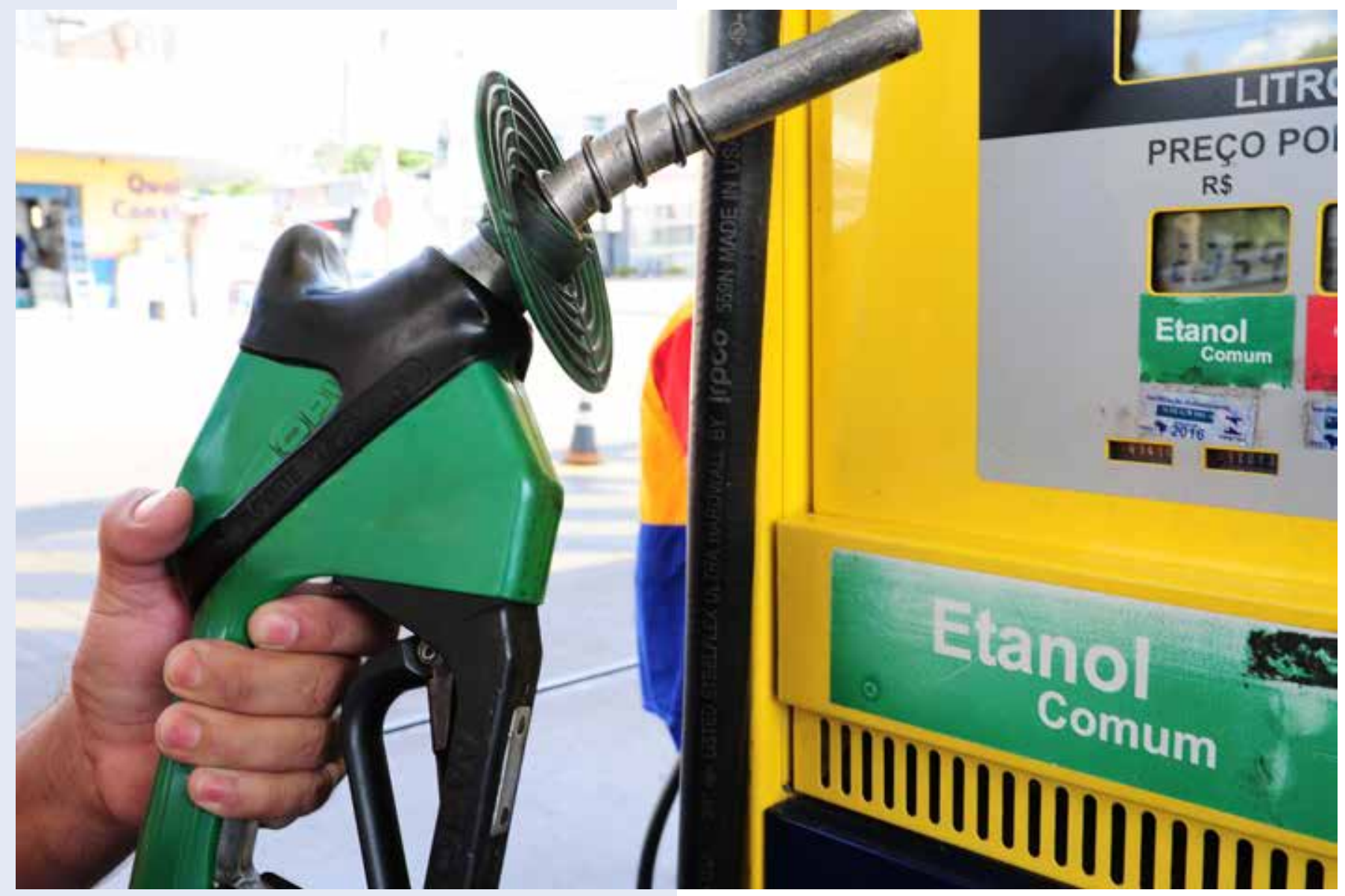




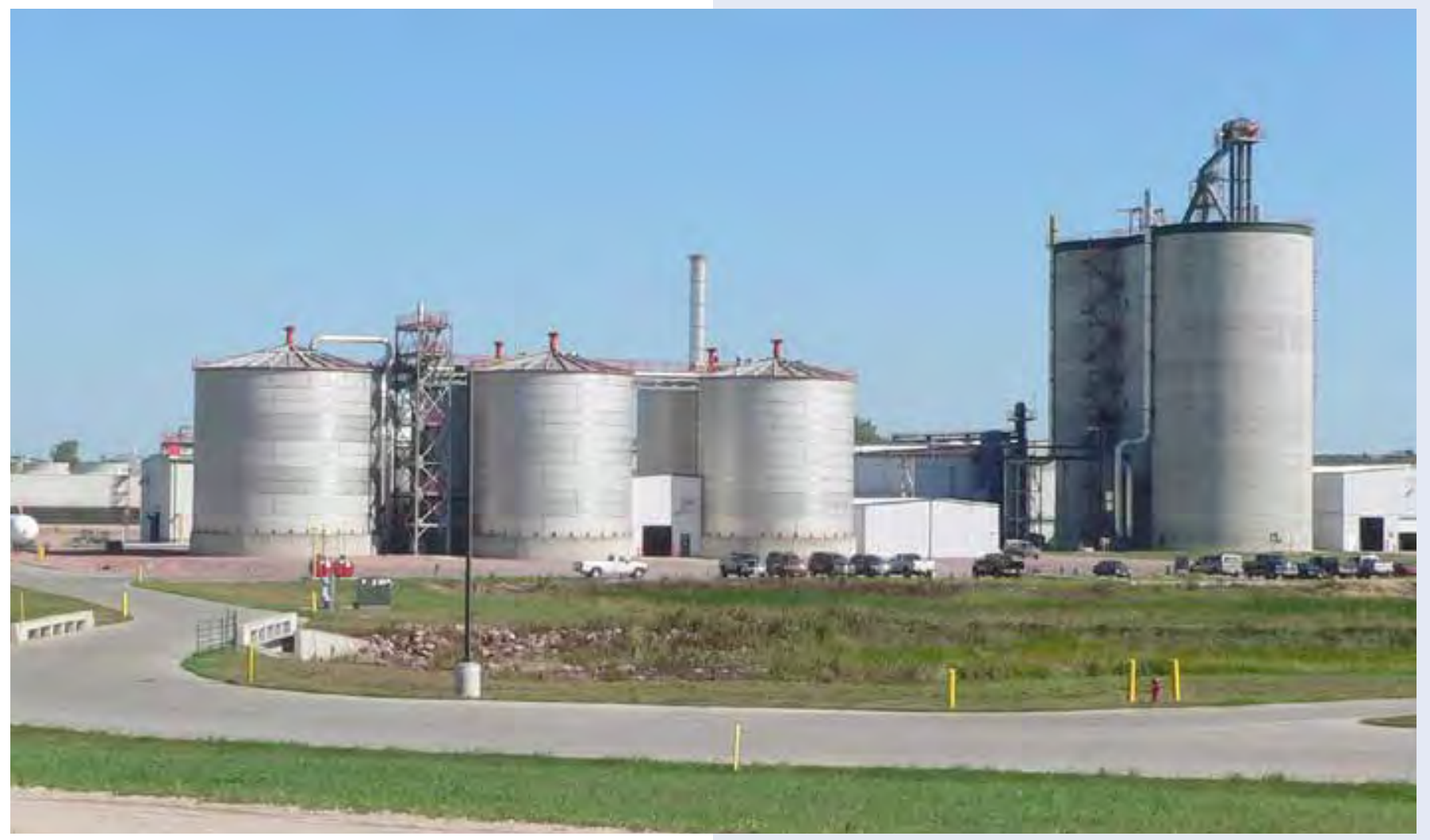

Tradicionalmente, la separación del sistema etanolagua se hace mediante destilación azeotrópica, utilizando benceno, pentano o dietil éter como solvente, o mediante destilación extractiva empleando etilenglicol o gasolina [6].

En este trabajo se propone la evaluación del material lignocelulósico C. ciliaris, como una materia prima potencial para la producción de etanol. Cenchrus ciliaris es un pasto silvestre conocido como zacate buffel que cubre el $8 \%$ de la superficie del Estado de Sonora, en el Noroeste de México. Dicha superficie corresponde aproximadamente a $1,850,000$ hectáreas, la mayoría concentradas en la parte central del Estado [7]. La composición típica de Cenchrus ciliaris incluye [8]: $88 \%$ de materia orgánica excluyendo proteínas, $10 \%$ de proteína cruda y $2 \%$ de cenizas insolubles en ácido. La materia orgánica está formada por $5.3 \%$ lignina; $35.3 \%$ celulosa; $31.8 \%$ hemicelulosa; y $15.6 \%$ otros materiales orgánicos no proteínicos.

Considerando la composición y naturaleza de Cenchrus ciliaris, éste representa un candidato potencial para la producción de etanol, ya que ofrece ventajas sobre otras materias primas. Algunas de estas ventajas incluyen su crecimiento en terrenos desérticos, bajos requerimientos de irrigación y alto rendimiento. Estos factores presentan efectos significativos sobre el ciclo de vida del proceso, el cual es un método para determinar los impactos ambientales durante el ciclo de vida completo de un producto y de su proceso de producción.

En el presente trabajo, se plantea como hipótesis que el uso de Cenchrus ciliaris como materia prima para producir etanol reduciría el consumo de energía y la emisión de gases de efecto invernadero con respecto a otros procesos convencionales para producción de etanol. Actualmente no existe antecedente en la literatura acerca de tal estudio, el objetivo de este trabajo fue determinar las características técnicas del proceso de producción de etanol a partir de Cenchrus ciliaris y realizar un análisis de su ciclo de vida con el fin de evaluar sus potenciales implicaciones ambientales.

\section{MÉTODO}

Para determinar las características técnicas relevantes y las implicaciones ambientales de la producción de etanol a partir de Cenchrus ciliaris, se integraron los resultados experimentales obtenidos en trabajos previos [9-11] e información relevante de la literatura [12-14]. Dicha información fue procesada para obtener indicadores cuantitativos. Las etapas consideradas en este estudio incluyeron: (a) preparación de la materia prima, (b) pretratamiento de Cenchrus ciliaris por hidrólisis ácidacuantificación de carbohidratos, (c) hidrólisis enzimática del residuo sólido, (d) fermentación de azúcares, y (e) análisis de ciclo de vida. Dichas etapas se detallan a continuación.

\section{Preparación de la Materia Prima}

La planta de Cenchrus ciliaris se recolectó manualmente en el ejido La Mora, Banamichi, Sonora, México, en la 
temporada de invierno del año 2012. La preparación de la materia prima recolectada en campo se realizó mediante secado a $80{ }^{\circ} \mathrm{C}$ durante $24 \mathrm{~h}$ en una mufla VWR Scientific modelo 1350 F. Una vez seca, el material se redujo de tamaño en un molino Pulvex 200 hasta tamaños de partícula menores de $0.18 \mathrm{~mm}$ de diámetro. Posteriormente se cribó y se seleccionaron dos fracciones de tamaño: la primera fracción incluyó partículas menores a $0.053 \mathrm{~mm}$; la segunda incluyó partículas en el intervalo de $0.147-0.173 \mathrm{~mm}$, con un tamaño promedio de $0.16 \mathrm{~mm}$. Ambas fracciones se sometieron a las etapas descritas a continuación.

\section{Pretratamiento por Hidrólisis ácida-cuantificación de carbohidratos (HPLC)}

Los experimentos de hidrólisis ácida se llevaron a cabo a en un reactor Parr de $2 \mathrm{~L}$ modelo 4850 a temperaturas de 120,135 y $150^{\circ} \mathrm{C}$, y concentraciones de ácido sulfúrico de 0.05 y $0.15 \mathrm{M}$. La masa inicial de biomasa utilizada en los experimentos fue de $10 \mathrm{~g}$ por litro de solución ácida. El tiempo de reacción fue de 60 minutos. Cada 10 minutos se recolectaron $5 \mathrm{~mL}$ de muestra de solución acuosa para su análisis. Las muestras fueron analizadas por HPLC para conocer la concentración de carbohidratos presentes en el producto de la hidrólisis. Para ello se utilizó una columna $\mathrm{Hi}$-Plex $\mathrm{H}$ marca Varian a $65^{\circ} \mathrm{C}$, que contiene una resina de intercambio catiónico que consiste de estireno sulfonado entrecruzado con copolímero de divinilbenceno. El diámetro de partícula del material de la columna es de
7-11 $\mu \mathrm{m}$. La fase móvil fue agua para HPLC marca Fermont H5052 suministrada a un flujo de $0.6 \mathrm{~mL} / \mathrm{min}$. Se utilizó un método isocrático, inyectándose 25 microlitros de muestra. Los detectores de índice de refracción Varian $\odot$ modelo Star 9020 y de UV-Visible Varian@ ProStar envían la señal eléctrica al integrador Shimadzu modelo Chromatopac C-R3A el cual registra en forma gráfica las especies químicas detectadas por el instrumento, generando un reporte del área bajo la curva obtenida. El detector de índice de refracción fue operado a $35^{\circ} \mathrm{C}$ y a $2.56 \times 10^{-6}$ RIU/F.S y el detector UV-visible trabajó a una longitud de onda de $254 \mathrm{~nm}$, para todas las muestras analizadas. Los estándares utilizados para las curvas de calibración fueron marca Sigma-Aldrich. Los tiempos de retención de los carbohidratos fueron 9, 10, 11 min para glucosa, xilosa y arabinosa, respectivamente.

La liberación de los monómeros de carbohidratos ocurre durante el pretratamiento de hidrólisis con ácido sulfúrico diluido a partir de la hemicelulosa contenida en Cenchrus ciliaris. A partir de un balance de masa para la hemicelulosa (ecuación 1) y para los carbohidratos en el reactor batch (ecuación 2) a volumen constante, con mezclado perfecto se obtuvieron las siguientes ecuaciones:

$$
\begin{gathered}
\frac{d H}{d t}=-k_{1}[H] \\
\frac{d C_{i}}{d t}=\alpha_{i} k_{1}[H]-k_{i}\left[C_{i}\right]
\end{gathered}
$$

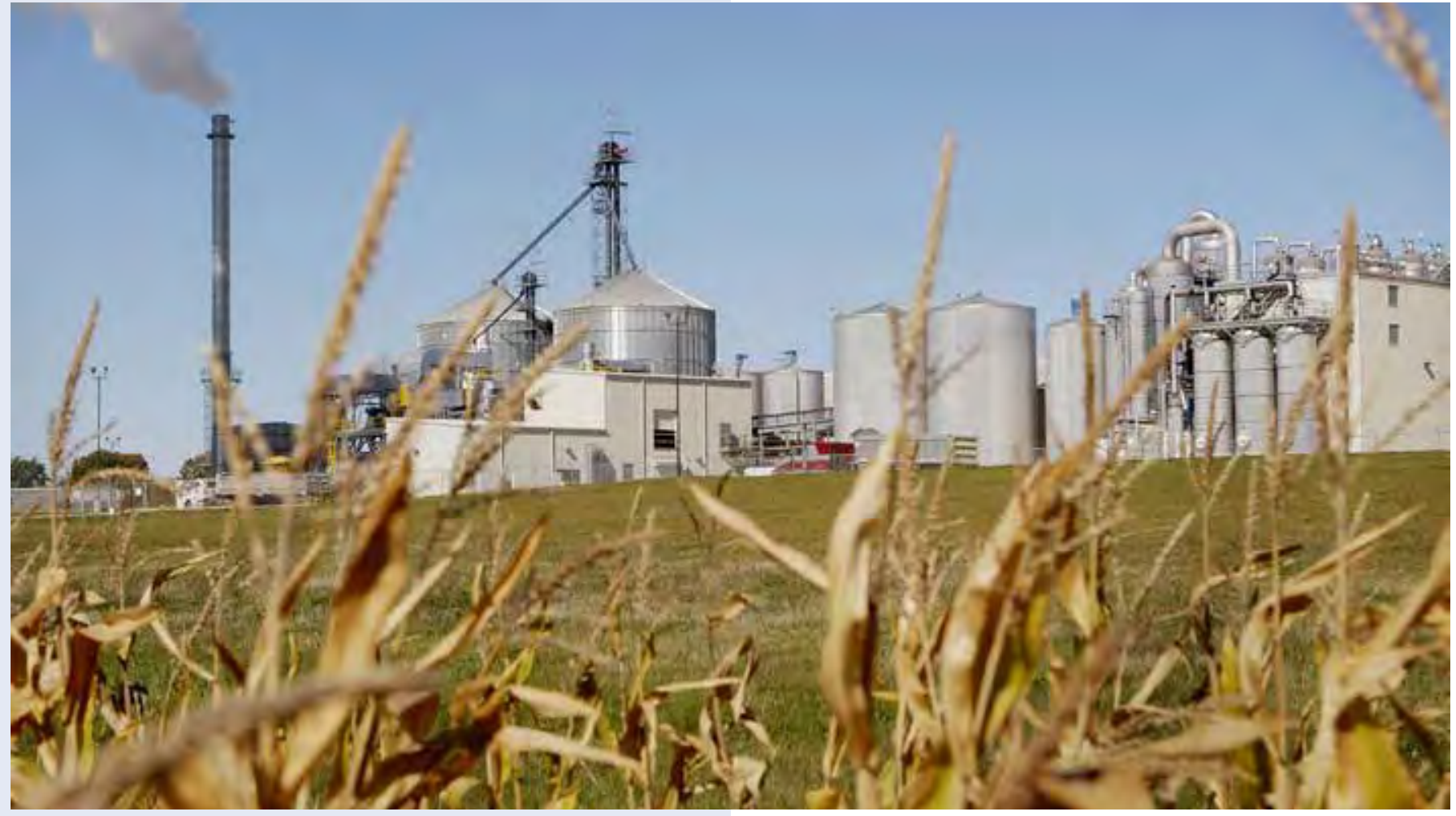




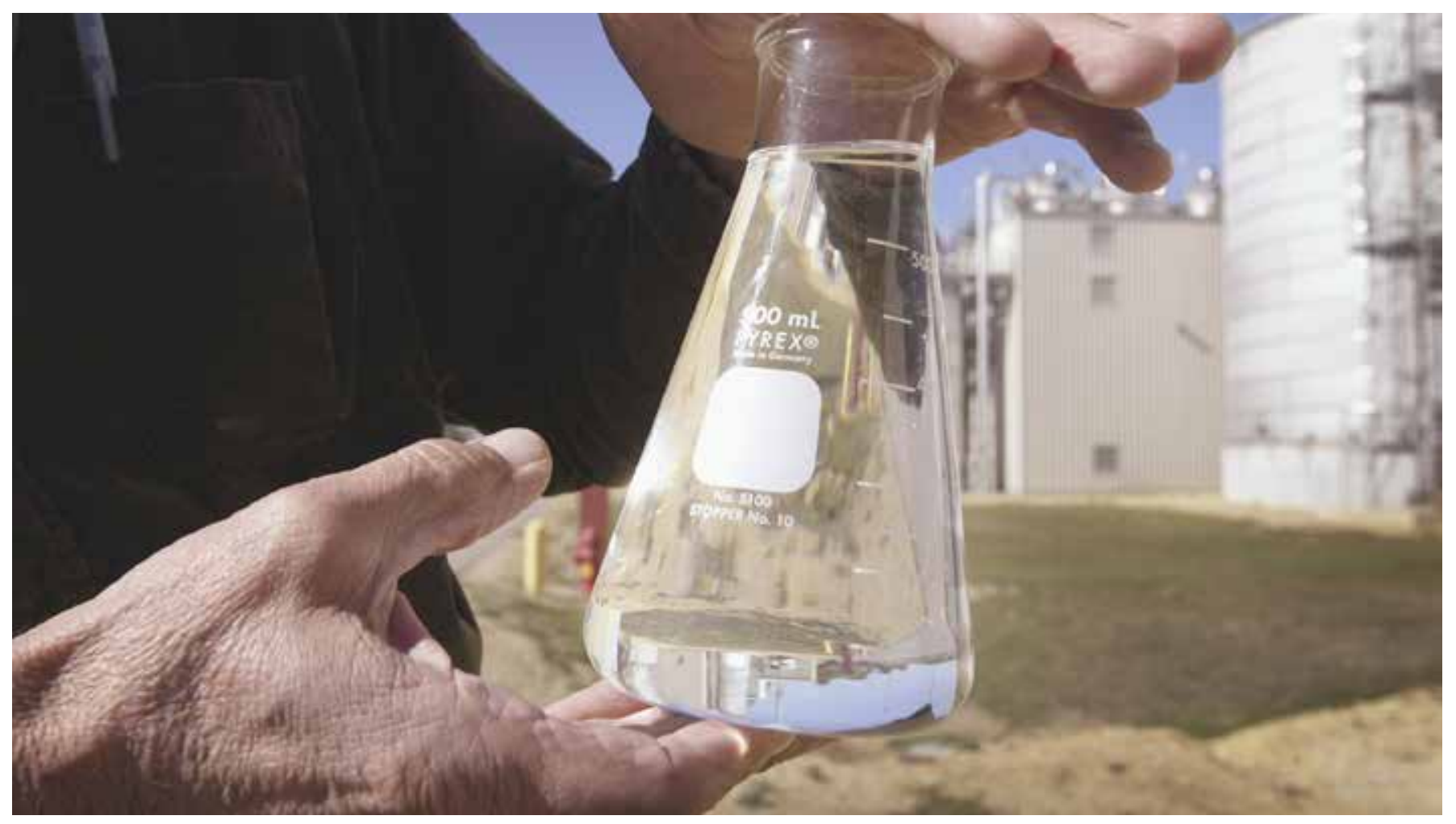

donde $H$ es la cantidad de hemicelulosa sólida por unidad de volumen de solución acuosa en el reactor $(\mathrm{mmol} / \mathrm{L}), C_{i}$ es la concentración del carbohidrato $i$ disuelto en la solución acuosa $(\mathrm{mmol} / \mathrm{L}), k$ es la constante cinética aparente de la descomposición de la hemicelulosa $\left(\min ^{-1}\right), k_{i}$ es la constante cinética de la degradación del carbohidrato $i\left(\min ^{-1}\right)$, y $a_{i}$ es el número de moles del carbohidrato $i$ producidas por mol de hemicelulosa consumida.

Se hace notar que en la ecuación (2) se ha supuesto que la reacción de degradación del carbohidrato $i$ en solución es irreversible y obedece a una cinética de primer orden con respecto a la concentración del carbohidrato disuelto $C_{i}$. Para un reactor isotérmico, los valores de las constantes cinéticas $\left(k_{1}, k_{i}\right)$ no dependen del tiempo. De acuerdo a las ecuaciones (1) y (2), si se conocen los coeficientes estequiométricos $\left(a_{i}\right)$ y los valores de $\mathrm{H}$ y $C_{i}$ a tiempo inicial $(\mathrm{t}=0)$, es posible calcular los valores de $H$ y $C_{i}$ en el reactor a cualquier tiempo. Durante los experimentos [9], se recolectaron datos de $C_{i}$ vs tiempo y se determinaron los coeficientes estequiométricos (ai) y los valores de $H$ y $\mathrm{C}_{\text {a }}$ tiempo inicial $(\mathrm{t}=0)$, Todo lo anterior, se estableció basados experimentos analizados mediante el optimizador Solver de la hoja electrónica Excel [15] se determinaron los valores de las constantes cinéticas $\left(k_{1}\right.$, $\left.k_{i}\right)$ y sus energías de activación aparentes a diferentes condiciones experimentales. Las ecuaciones (1) y (2) ajustaron aceptablemente los datos experimentales con un valor del factor de correlación promedio $r^{2}=0.9889$.

A fin de generalizar los resultados, la constante $k_{1}$ se correlacionó con la temperatura absoluta mediante la ecuación de Arrhenius:

$$
k_{1}=A \exp \left(-\frac{E_{a}}{R T}\right)
$$

donde $E_{a}$ es la energía de activación aparente $(\mathrm{kJ} / \mathrm{mol})$, $A$ es el factor preexponencial $\left(\mathrm{min}^{-1}\right), R$ es la constante universal de los gases $(\mathrm{kJ} / \mathrm{molK})$ y $T$ es la temperatura absoluta (K).

\section{Hidrólisis Enzimática}

A partir del pretratamiento ácido de Cenchrus ciliaris se obtuvo un residuo sólido en forma de fibras, el cual se hidrolizó enzimáticamente con celulasas comerciales obtenidas por Aspergillus niger (USB Corporation, Cleveland, $\mathrm{OH}$ USA). Para la reacción enzimática se utilizaron $0.5 \mathrm{~g}$ de fibras en un volumen de $50 \mathrm{~mL}$ de agua destilada con las condiciones óptimas de la enzima $(\mathrm{pH}$ 4.5 y $40^{\circ} \mathrm{C}$ ) y diferentes relaciones enzima-sustrato $(0.02-$ $3.2 \mathrm{~g} / \mathrm{L}$ ). La hidrólisis enzimática inicia al agregar la enzima en el sistema de reacción, tomando $1 \mathrm{~mL}$ de muestra cada 20 minutos durante 2 horas [10]. Los carbohidratos se cuantificaron con la técnica de HPLC descrita en el apartado anterior.

Para describir la cinética de las reacciones enzimáticas se utilizó el modelo de Michaelis-Menten, ecuación (4):

$$
V=\frac{V_{\max }(E)}{K_{m}+E}
$$

donde $\mathrm{V}$ es la rapidez de producción de glucosa por 


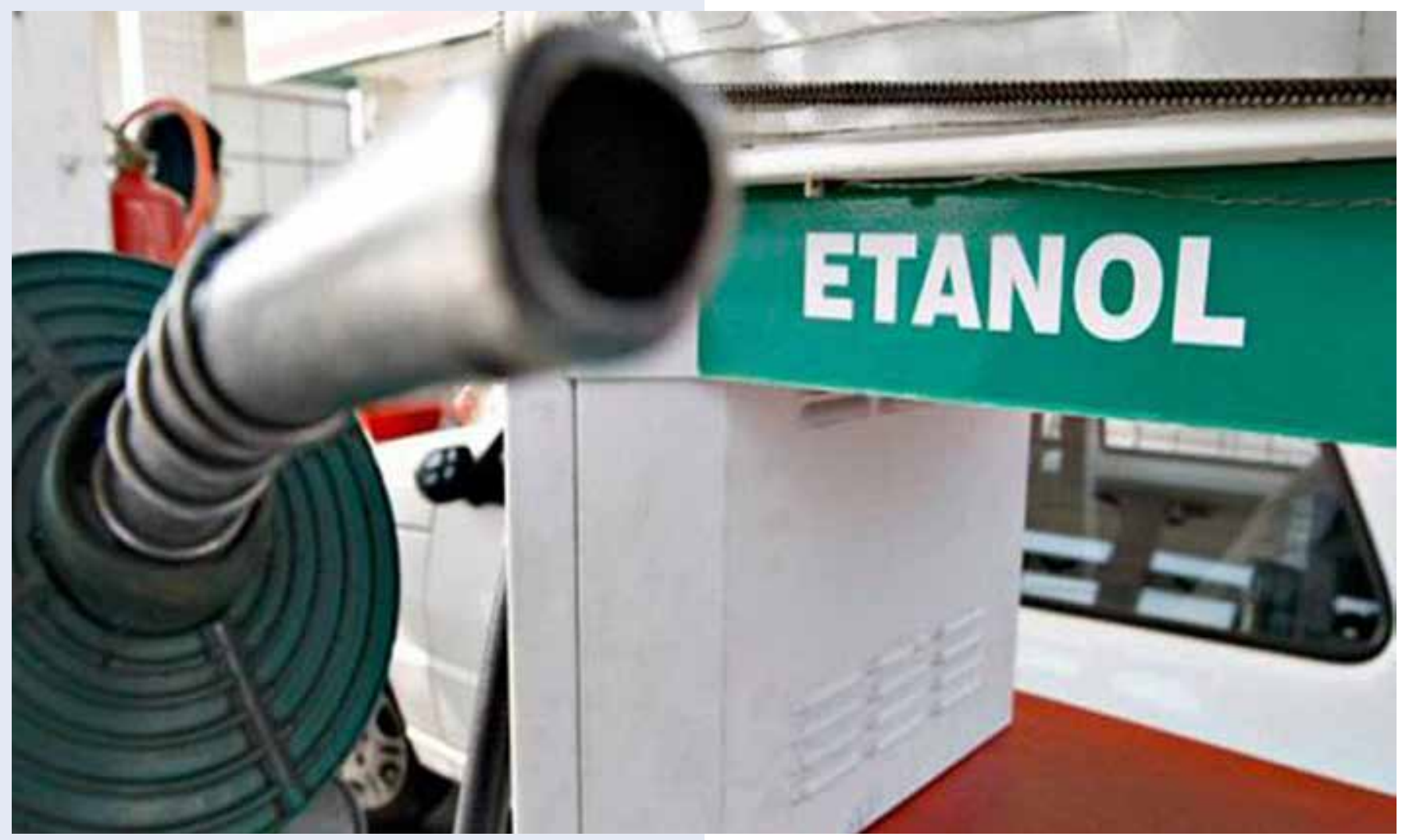

hidrólisis de celulosa ( $\mathrm{g} / \mathrm{Lmin}), \mathrm{V}_{\max }$ es la máxima rapidez de producción de glucosa por hidrólisis de celulosa a la temperatura y $\mathrm{pH}$ del sistema $(\mathrm{g} / \mathrm{Lmin})$, E es la relación enzima-sustrato $(\mathrm{g} / \mathrm{L})$ y $\mathrm{K}_{\mathrm{m}}$ es el valor de la relación enzima-sustrato $(\mathrm{g} / \mathrm{L})$ para la cual se cumple que: $\mathrm{V}=(1 / 2) \mathrm{V}_{\max }$. Los valores de $\mathrm{K}_{\mathrm{m}}$ y $\mathrm{V}_{\text {max }}$ se obtuvieron mediante regresión no lineal de los datos experimentales de $V$ vs E por la técnica de mínimos cuadrados [16].

\section{Fermentación}

Cada fermentación fue realizada en un matraz Kitazato conteniendo $1 \mathrm{~L}$ de solución por gramo de levadura, conectado a una trampa de agua. Para la fermentación de carbohidratos se utilizó una levadura de Saccharomyces cerevisiae y un medio ácido de nutrientes para levadura $\left(0.46 \mathrm{~g}\right.$ de $\mathrm{CO}\left(\mathrm{NH}_{2}\right)_{2}, 0.61 \mathrm{~g}$ de $\mathrm{K}_{2} \mathrm{HPO}_{4}$ ). La fermentación fue realizada dentro de una incubadora a una temperatura de $30{ }^{\circ} \mathrm{C}$ por periodos de $36-42$ horas. Se determinó etanol como producto de la fermentación por medio de HPLC descrita con detalle en la sección de hidrólisis ácida [11]. El tiempo de retención del etanol fue de $29 \mathrm{~min}$.

\section{Análisis de Ciclo de Vida}

En este análisis se comparan la energía necesaria y las emisiones de gases de efecto invernadero para la obtención de combustibles que actualmente se producen como gasolina, etanol de maíz y etanol de materiales lignocelulósicos. Estas referencias permiten estimar la energía necesaria así como las emisiones de gases en la producción de etanol a partir de Cenchrus ciliaris.

El esquema global para la producción de etanol a partir de Cenchrus ciliaris se muestra en la Figura 1. El esquema toma en cuenta la maquinaria, transporte, electricidad y el combustible utilizado, así como la gestión de residuos durante el proceso. Durante la etapa de cultivo es necesario considerar la preparación del suelo, del campo, y la clasificación y empaque para la obtención de un alto rendimiento de la cosecha. En la etapa de conversión de etanol, se realizaron estudios a escala laboratorio para encontrar las condiciones óptimas para los procesos de pretratamiento, hidrólisis y fermentación. Después de la recuperación del etanol, en la etapa 3 se consideran los almacenamientos de gasolina y etanol para hacer una mezcla final que es distribuida a las estaciones de servicio para el cliente final. 

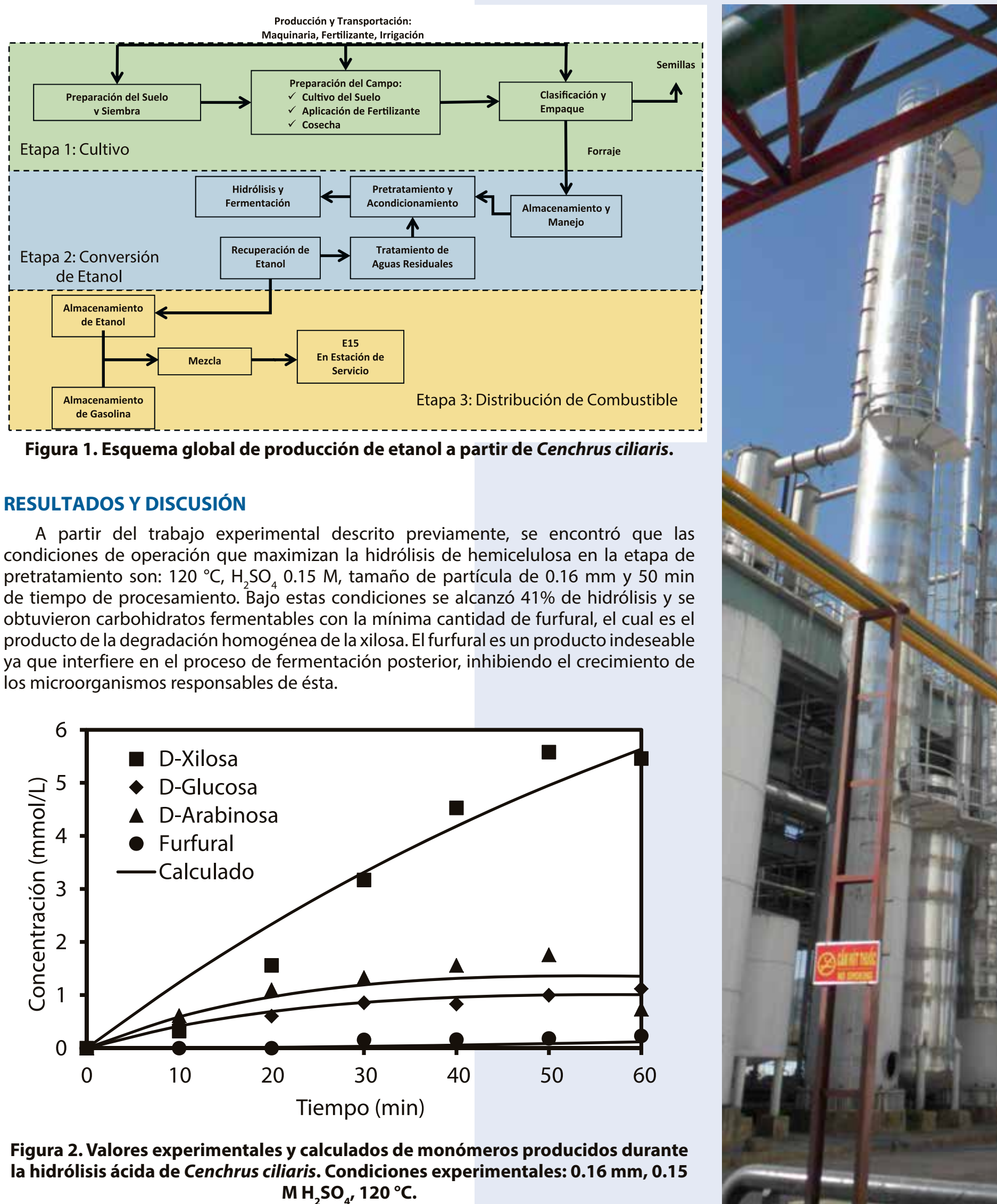

Figura 2. Valores experimentales y calculados de monómeros producidos durante la hidrólisis ácida de Cenchrus ciliaris. Condiciones experimentales: $0.16 \mathrm{~mm}, 0.15$ $\mathrm{M} \mathrm{H}_{2} \mathrm{SO}_{4^{\prime}} 120^{\circ} \mathrm{C}$. 


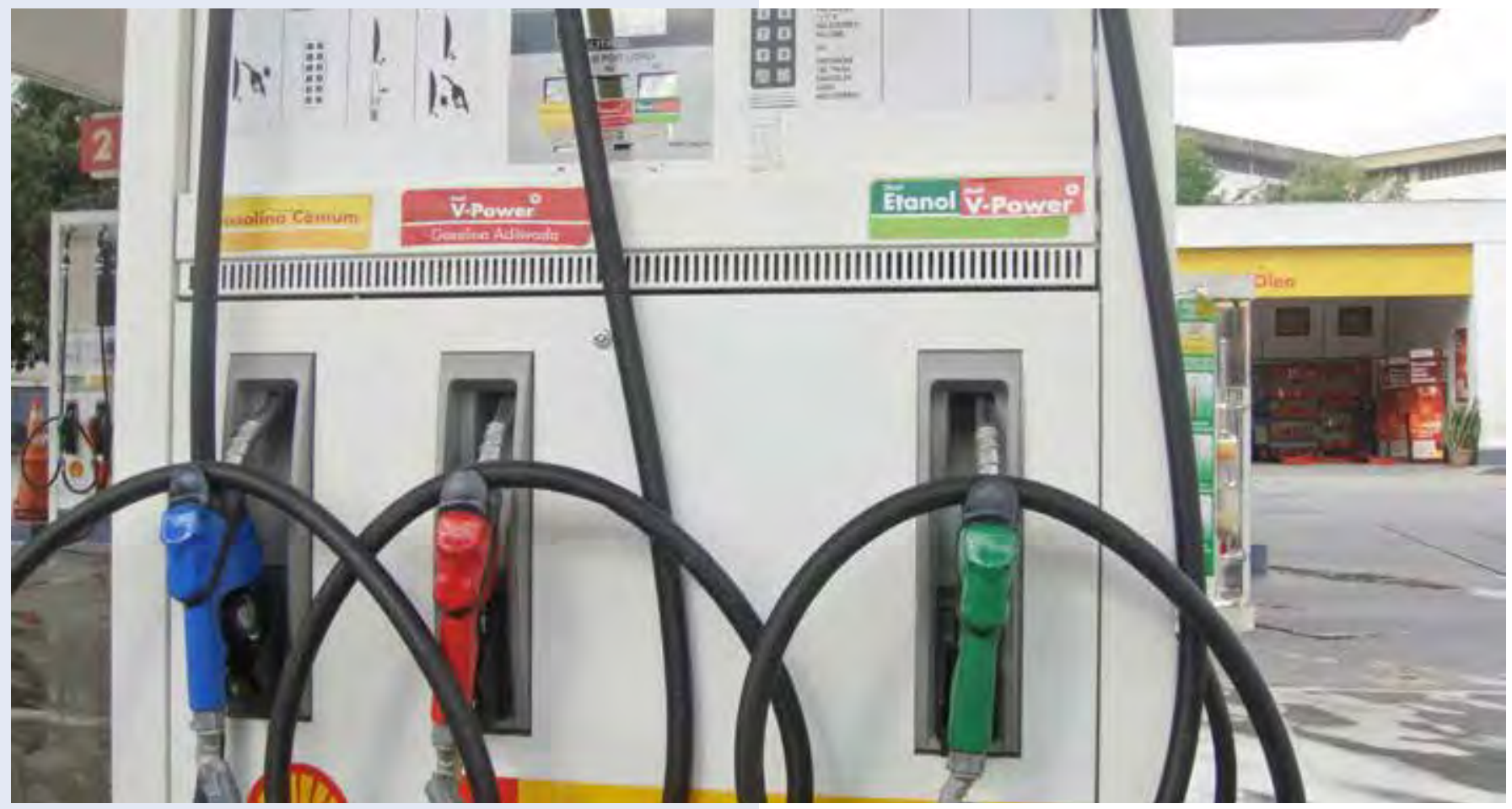

A partir de las ecuaciones (1) y (2) y el tratamiento de los datos experimentales de la hidrólisis ácida se determinaron los valores de la constante cinética $k$, para cada condición experimental. La Figura 2 muestra los valores de la concentración de carbohidratos experimentales y los calculados por las ecuaciones (1) y (2) para las condiciones experimentales: $0.16 \mathrm{~mm}, 0.15 \mathrm{M}$ de $\mathrm{H}_{2} \mathrm{SO}_{4}$ y $120{ }^{\circ} \mathrm{C}$. En general, se obtuvo una buena concordancia de las predicciones del modelo con los datos cinéticos. La Tabla 1 resume los resultados obtenidos.

Tabla 1. Valores de la energía de activación aparente y factor preexponencial para la constante cinética de hidrólisis de hemicelulosa de Cenchrus ciliaris, $\boldsymbol{k}_{1}$.

\begin{tabular}{|l|l|l|l|}
\hline $\begin{array}{c}\text { Tamaño de } \\
\text { partícula } \\
(\mathrm{mm})\end{array}$ & $\begin{array}{c}\text { Concentración } \\
\text { de } \mathrm{H}_{2} \mathrm{SO}_{4}(\mathrm{M})\end{array}$ & $\begin{array}{c}\mathrm{Ea} \\
(\mathrm{kJ} / \mathrm{mol})\end{array}$ & \multicolumn{1}{|c|}{$\left(\mathrm{min}^{-1}\right)$} \\
\hline$<0.053$ & 0.05 & 105 & $1.0 \times 10^{12}$ \\
\hline$<0.053$ & 0.15 & 19 & 9.48 \\
\hline 0.16 & 0.05 & 64 & $5.4 \times 10^{6}$ \\
\hline 0.16 & 0.15 & 11 & 1.12 \\
\hline
\end{tabular}

La Figura 3 muestra los valores experimentales y calculados de la velocidad de producción de glucosa durante la hidrólisis enzimática con celulasas de Aspergillus niger en función de la relación enzima-sustrato. Mediante el procedimiento descrito previamente se obtuvieron los valores de las constantes cinéticas: $K_{m}=1.40 \mathrm{~g} / \mathrm{L}$ y $V_{\text {max }}=0.019$ $\mathrm{g} /($ Lmin). Se observa que el modelo de Michaelis-Menten (ecuación 3) describe satisfactoriamente la cinética de la hidrólisis enzimática en todo el intervalo experimental.

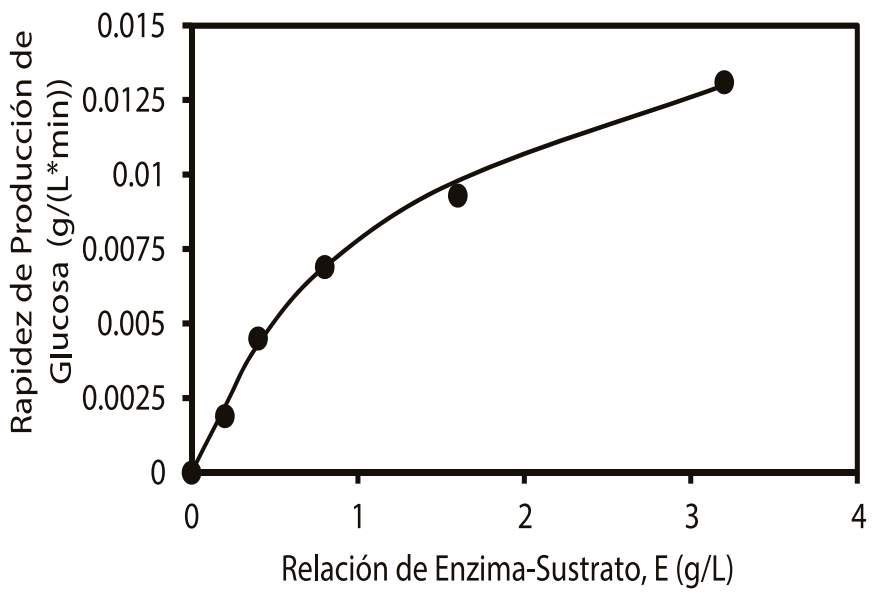

Figura 3. Valores experimentales y calculados de la rapidez de producción de glucosa durante la hidrólisis enzimática con celulasas de Aspergillus niger.

La relación enzima-sustrato óptima para la hidrólisis enzimática de la celulosa del residuo sólido obtenido del pretratamiento ácido fue de $3.2 \mathrm{~g} / \mathrm{L}$, obteniéndose $54 \%$ de hidrólisis de celulosa en 120 minutos de reacción.

Para la estimación del rendimiento teórico de producción de etanol se consideró como base de cálculo una tonelada de zacate buffel, considerando la composición y los factores metabólicos de conversión de los carbohidratos producidos. Los factores de conversión utilizados incluyen: $0.51 \mathrm{~kg}$ de etanol por $\mathrm{kg}$ de carbohidrato 
consumido. La producción de etanol estimada fue de $422 \mathrm{~L}$ por tonelada de Cenchrus ciliaris.

A nivel laboratorio, al fermentar los productos de la hidrólisis ácida se obtuvo un rendimiento experimental correspondiente a $80 \mathrm{~L}$ por tonelada de zacate buffel, lo cual es posible incrementar aplicando el paso de hidrólisis enzimática de la celulosa. Analizando la concentración inicial de carbohidratos en el sistema de fermentación, se estima que la cantidad de etanol producido en la fermentación es de $2.5 \mathrm{~g} / \mathrm{L}$, la diferencia es atribuida al efecto del furfural, el cual inhibe el crecimiento del microorganismo responsable de la fermentación.

La Figura 4 muestra una comparación de la energía derivada del petróleo que es consumida para producir 1 MJ de combustible de diferente origen. La información de dicha gráfica procede de la literatura [12-14]. Se hace notar que la producción de etanol a partir de maíz y materiales lignocelulósicos consume aproximadamente $60 \%$ menos energía derivada del petróleo que la utilizada para producir gasolina.

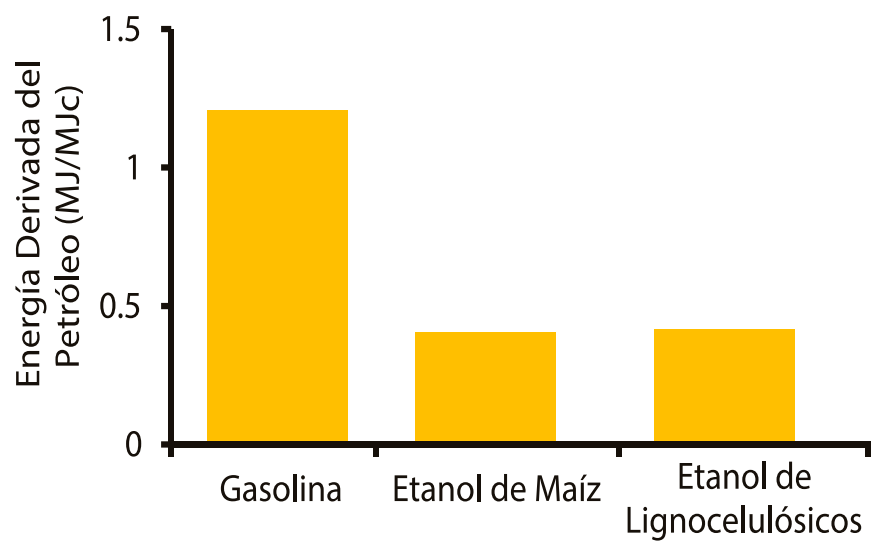

Combustible

Figura 4. Energía derivada del petróleo requerida para producción de combustibles a partir de diferentes materias primas [12-14].
Similarmente, la Figura 5 presenta la comparación de las emisiones de gases de efecto invernadero en gramos de $\mathrm{CO}_{2}$ equivalentes por $\mathrm{MJ}$ de combustible producido. Se observa que la producción de etanol de maíz emite tanto $\mathrm{CO}_{2}$ como la producción de gasolina. Asimismo, las emisiones de $\mathrm{N}_{2} \mathrm{O}$ durante la cosecha y recolección del maíz y de los residuos lignocelulósicos, son mayores que durante la producción de gasolina. Sin embargo, el etanol derivado de lignocelulósicos reduce las emisiones de gases de efecto invernadero en $40 \%$ y $45 \%$ comparado con la gasolina y el etanol de maíz, respectivamente.

En función de sus características físicas y químicas, cabe esperar que el procesamiento a nivel industrial de Cenchrus ciliaris presente un comportamiento similar al mostrado en las Figuras 4 y 5 para otros materiales lignocelulósicos reportados en la literatura. Esto lo convierte en una materia prima factible para la producción de etanol por vías biotecnológicas.

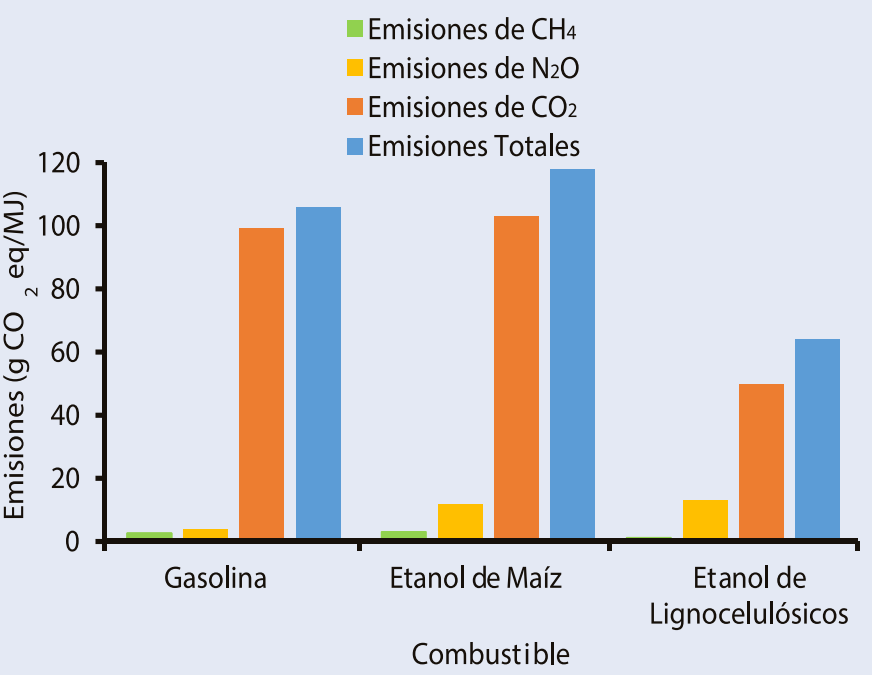

Figura 5. Emisiones de gases de efecto invernadero de combustibles [12-14].

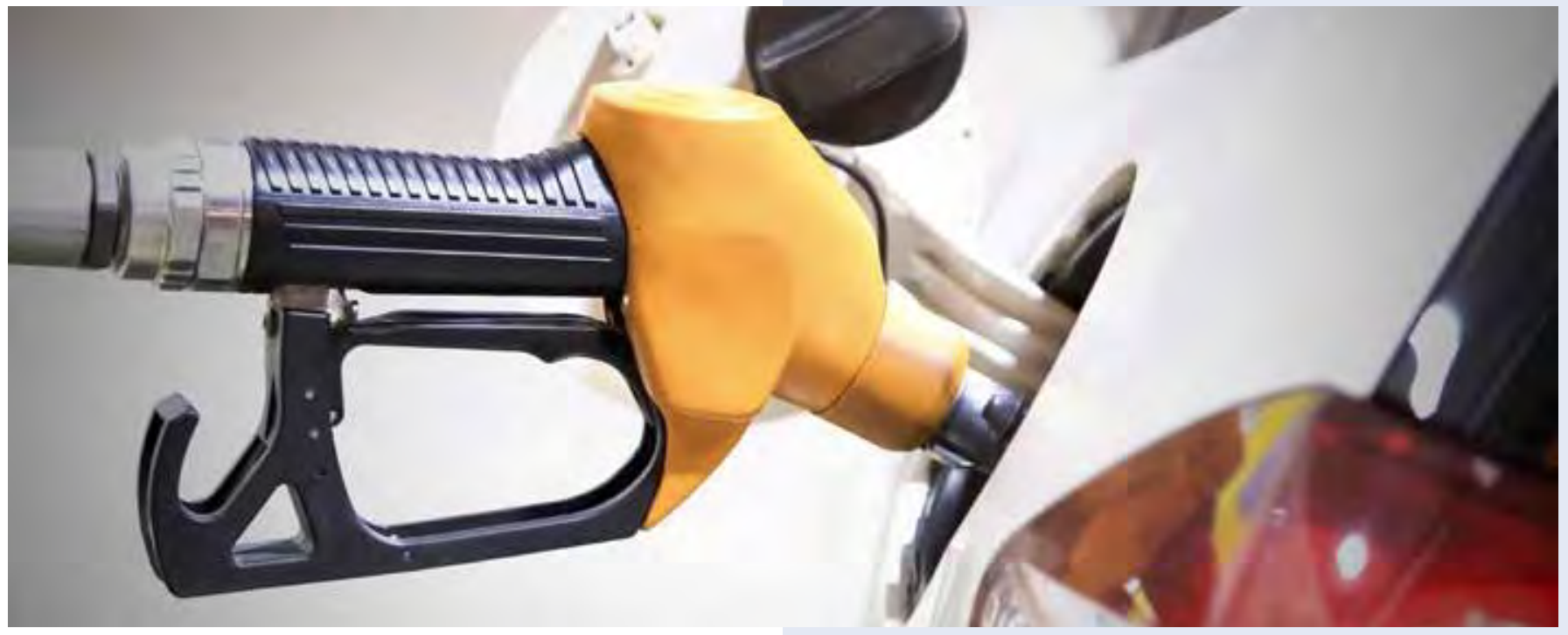




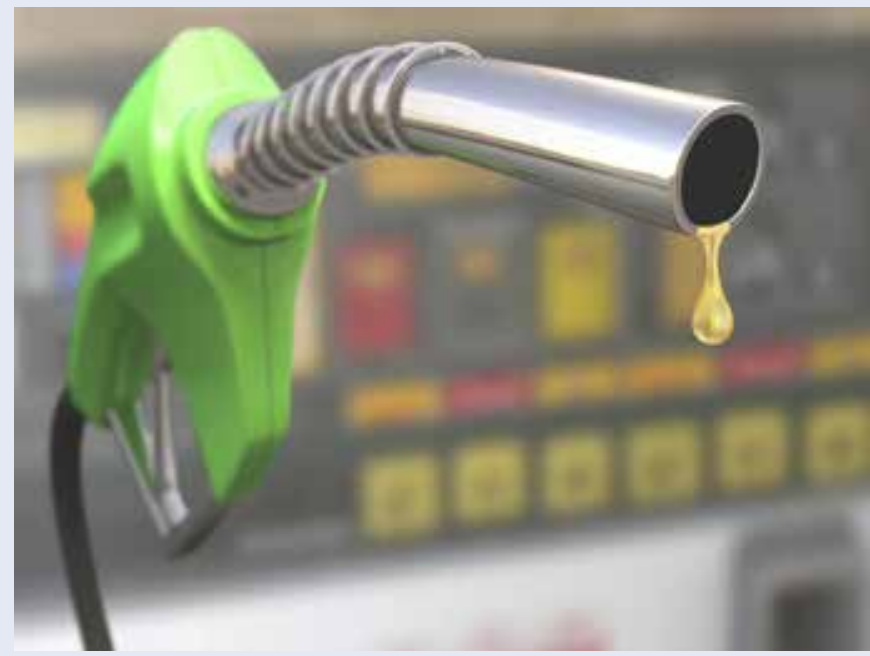

\section{CONCLUSIÓN}

En el presente trabajo se caracterizaron a nivel laboratorio las etapas de producción de etanol a partir de Cenchrus ciliaris: pretratamiento ácido, hidrólisis enzimática y fermentación. Los resultados de este estudio indican que la biomasa obtenida a partir de Cenchrus ciliaris presenta potencial como materia prima para la producción de etanol. Basado en la literatura, dicho potencial se fundamenta en rendimientos técnicos del proceso, así como en la posibilidad de reducir la emisión de gases de efecto invernadero a la atmósfera y el consumo de energía derivada del petróleo por MJ de combustible producido.

\section{BIBLIOGRAFÍA}

[1] M. Taherzadeh, and K. Karimi. "Acid-Based Hydrolysis Processes for Ethanol from Lignocellulosic Materials: A review". Bioresources. Vol. 2(3), pp. 472-499, 2007.

[2] N. Sarkar, S. Kumar, S. Bannerjee, K. Aikat. "Bioethanol production from agricultural wastes: An overview". Renewable Energy, vol. 37, pp. 19-27, 2012.

[3] S. Kim, and B. Dale. "Global potential bioethanol production from wasted crops and crop residues". Biomass Bioenerg. Vol. 26(4), pp. 361-375, 2004.

[4] C. Wyman, B. Dale, R. Elander, M. Holtzapple, M. Ladisch, Y. Lee. "Coordinated development of leading biomass pretreatment technologies", Bioresource Technology, vol. 96. pp. 19591966, 2005.

[5] S. Pingali, V. Urban, W. Heller, J. McGaughey, H. O'Neill, M. Foston, D. Myles, A. Ragauskas, B. Evans. "Breakdown of Cell Wall Nanostructure in Dilute Acid Pretreated Biomass", Biomacromolecules, vol. 11. pp. 2329-2335, 2010.

[6] C. Vásquez, C. Ruiz, D. Arango. "Production of anhydrous ethanol by extractive distillation with salt effect". Dyna rev. fac. nac. minas. Vol. 74(151), pp. 53-59, 2007.

[7] K. Franklin, K. Lyons, P. Nagler, D. Lampkin, E. Glenn, F. MolinaFreaner, T. Markow, and A. Huete. "Buffelgrass (Pennisetum ciliare) land conversion and productivity in the plains of Sonora, Mexico". Biological Conservation. vol. 127(1), pp. 6271, 2006.

[8] R. Ramírez, A. Enríquez, F. Lozano. "Valor nutricional y degradabilidad ruminal del zacate buffel y nueve zacates nativos del NE de México". CIENCIA UANL / vol. IV, No. 3, 2001.

[9] K. Encinas. "Estudio Cinético de la Formación de Xilosa por
Hidrólisis con Ácido Sulfúrico Diluido de Cenchrus ciliaris". Tesis de Maestría. Universidad de Sonora, 2010.

[10] J. Flores. "Estudio Cinético de la Hidrólisis enzimática de Tejidos Pretratados con Ácido Sulfúrico Diluido de Cenchrus ciliaris". Tesis de Licenciatura. Universidad de Sonora, 2010.

[11] J. Torrescano. "Obtención de Etanol a partir de Buffel". Tesis de Licenciatura. Universidad de Sonora, 2009.

[12] N. Brinkman, M. Wang, T. Weber, and T. Darlington. Well-towheels analysis of advanced fuel/vehicle systems -A North American study of energy use, greenhouse gas emission, and criteria pollutant emission, General Motors CorporationArgonne National Laboratory-Air Improvement Resources, Inc. 238, 2005.

[13] MA. Delucchi. "A multi-country analysis of lifecycle emissions from transportation fuels and motor vehicles". Institute of Transportation Studies. University of California, Davis UCDITS-RR-05-10, 2005.

[14] J. Sheehan, V. Camobreco, J. Duffield, M. Graboski, and H. Shapouri. "Life cycle inventory of biodiesel and petroleum diesel for use in an urban bus". National Renewable Energy Laboratory. NREL/SR-580-24089, 1998.

[15] Microsoft Excel, 2013.

[16] M. Córdova. "Estadística Descriptiva e Inferencial, Aplicaciones". Quinta Edición. MOSHERA, 2003. https:// en.wikipedia.org/wiki/Cosmic_microwave_background.

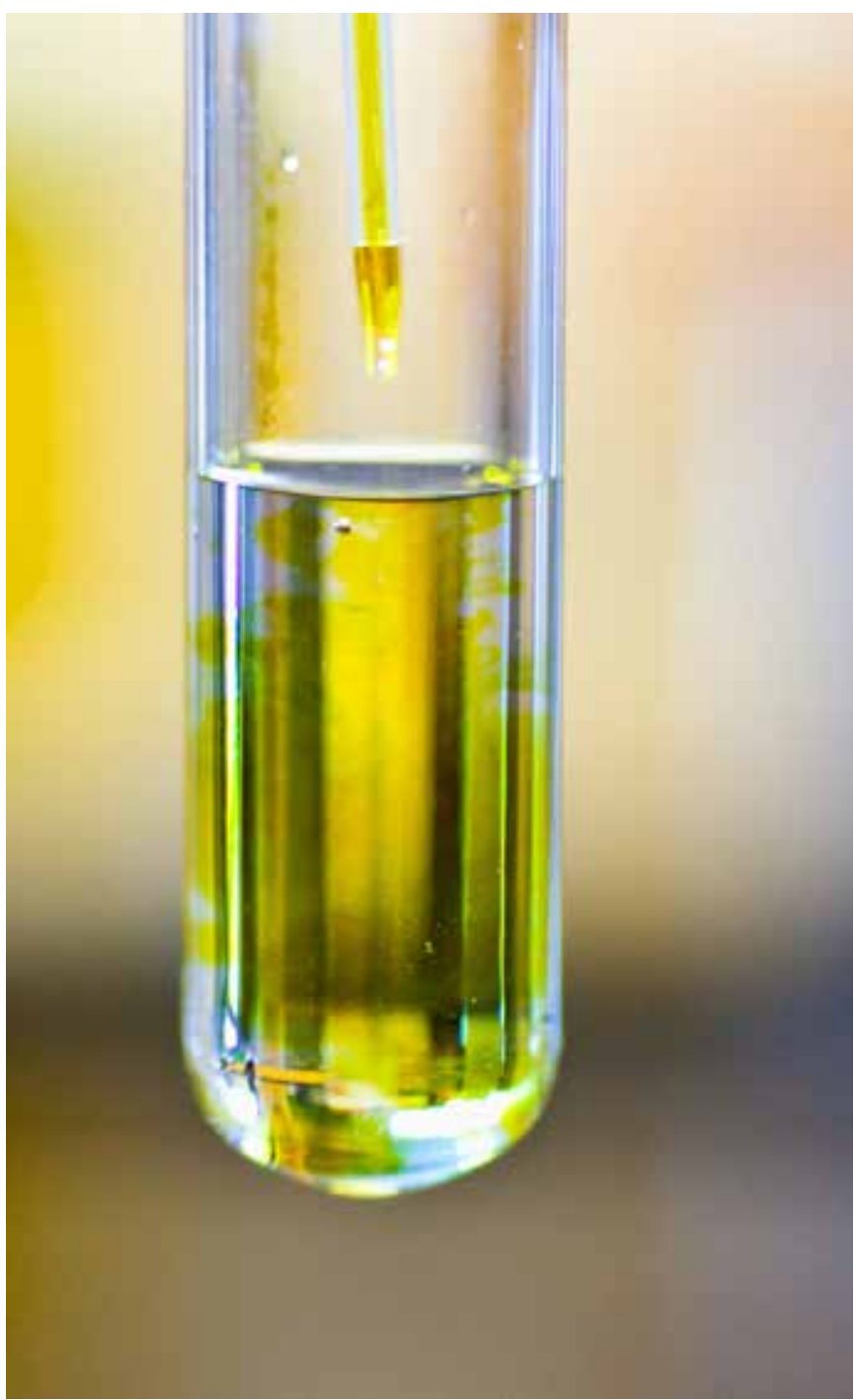

